\title{
Taspase1 orchestrates fetal liver hematopoietic stem cell and vertebrae fates by cleaving TFIIA
}

\author{
Hidetaka Niizuma, ${ }^{1,2}$ Adam C. Searleman, ${ }^{3}$ Shugaku Takeda, ${ }^{1}$ Scott A. Armstrong, ${ }^{4}$ \\ Christopher Y. Park, ${ }^{5}$ Emily H. Cheng, ${ }^{1,6}$ and James J. Hsieh ${ }^{3}$ \\ ${ }^{1}$ Human Oncology \& Pathogenesis Program, Memorial Sloan Kettering Cancer Center, New York, New York, USA \\ 2Department of Pediatrics, Tohoku University School of Medicine, Sendai, Japan. ${ }^{3}$ Department of Medicine, Washington \\ University School of Medicine, St. Louis, Missouri, USA. ${ }^{4}$ Department of Pediatric Oncology, Dana-Farber Cancer Institute, \\ Boston, Massachusetts, USA. ${ }^{5}$ Department of Pathology, NYU School of Medicine, New York, New York, USA. ${ }^{6}$ Department \\ of Pathology, Memorial Sloan Kettering Cancer Center, New York, New York, USA.
}

Taspase1, a highly conserved threonine protease encoded by TASP1, cleaves nuclear histonemodifying factors and basal transcription regulators to orchestrate diverse transcription programs. Hereditary loss-of-function mutation of TASP1 has recently been reported in humans as resulting in an anomaly complex syndrome, which manifests with hematological, facial, and skeletal abnormalities. Here, we demonstrate that Taspase1-mediated cleavage of TFIIA $\alpha-\beta$, rather than of MLL1 or MLL2, in mouse embryos was required for proper fetal liver hematopoiesis and correct segmental identities of the axial skeleton. Homozygous genetic deletion of Taspase1 disrupted embryonic hematopoietic stem cell self-renewal and quiescence states and axial skeleton fates. Strikingly, mice carrying knockin noncleavable mutations of TFIIA $\alpha-\beta$, a well-characterized basal transcription factor, displayed more pronounced fetal liver and axial skeleton defects than those with noncleavable MLL1 and MLL2, 2 trithorax group histone H3 trimethyl transferases. Our study offers molecular insights into a syndrome in humans that results from loss of TASP1 and describes an unexpected role of TFIIA $\alpha-\beta$ cleavage in embryonic cell fate decisions.

Conflict of interest: $\mid$ JH has received consulting fees from Eisai and BostonGene; clinical trial funding from Bristol Myers Squibb, Merck USA AstraZeneca, Exelixis, Calithera, and Sillajen; and research funding from Merck USA, BostonGene, and TScan. JHH has equity share in BostonGene.

Copyright: ( 2021 , Niizuma et al. This is an open access article published under the terms of the Creative Commons Attribution 4.0 International License.

Submitted: March 8, 2021

Accepted: June 17, 2021

Published: August 9, 2021

Reference information: /CI Insight. 2021;6(15):e149382.

https://doi.org/10.1172/jci. insight.149382.

\section{Introduction}

Recently, a novel human hereditary anomaly syndrome was recognized in association with loss-of-function mutations in the TASP1 gene (1-3). Those patients were characterized with microcephaly, developmental delay, distinctive facial features, and other anomalies, including anemia, thrombocytopenia, and lymphocytopenia $(2,3)$. TASP1 codes for Taspase1, which is an evolutionarily conserved threonine protease that cleaves and regulates nuclear proteins, most notably, MLL (KMT2A, also known as MLL1) and TFIIA $\alpha-\beta$ (4-6). Taspase 1 is a $50 \mathrm{kD} \alpha-\beta$ proenzyme that undergoes intramolecular autoproteolysis to produce mature, active $\alpha 28 / \beta 22$ heterodimeric protease $(4,6-8)$. Cleavage of Taspase 1 substrates occurs distal to the aspartate residue within the conserved IXQL(V)D/G motif $(4,9)$

The bona fide Taspase1 substrates are MLL1, MLL2 (also known as KMT2B), TFIIA $\alpha-\beta$ (also known as GTF2A1), ALF (TFIIA-like factor, also known as GTF2A1L) and Drosophila HCF (dHCF) (4, 5, 10 12). MLL1, a member of the trithorax group (Trx-G) epigenetic modifiers, is a histone methyl transferase (HMT) that trimethylates $\mathrm{H} 3$ at lysine 4, generating a histone mark of active transcription $(13,14)$. In the absence of proteolytic activation, immature MLL1 polypeptide displays reduced HMT activity and, hence, functions as a hypomorphic mutant (11). TFIIA, comprising 3 polypeptides ( $\alpha, \beta$, and $\gamma)$, complexes with TATA-binding protein (TBP) and constitutes an integral part of the basal transcription machinery (15-19). Precursor TFIIA $\alpha-\beta$, encoded by a single gene, Gtf2a1, is processed into TFIIA $\alpha$ and $-\beta$ subunits by Taspase 1 -mediated cleavage (5). Complexes of TFIIA $\alpha-\beta / \gamma$ containing noncleaved TFIIA $\alpha-\beta$ have transcriptional activity in vitro, and its maturation into TFIIA $\alpha / \beta / \gamma$ increases its susceptibility to regulatory degradation (5). In addition, TFIIA $\alpha-\beta / \gamma$ and TFIIA $\alpha / \beta / \gamma$ are different in the pattern of interactions with TFIID, an important factor for promoter recognition (20). Since TBP-like protein was reported to be a negative regulator of the Taspase1-mediated processing of TFIIA, Taspase1 may fine-tune the transcription of 
genes through these factors (21). Previous studies revealed that the proteolytic cleavage of TFIIA is critical for male spermiogenesis (22) and craniofacial development (23).

Taspase1-mediated cleavage of each substrate may account for various phenotypes of Taspase1 deficiency, or, alternatively, protease-independent biological activities of Taspase1 may exist. Here, we performed mouse genetic studies to interrogate the biological significance of cleavage of MLL1, MLL2, and TFIIA. Taspase1-mediated cleavage of TFIIA has the most prominent effects on fetal hematopoiesis as well as on the specification of axial skeleton, offering molecular insights concerning a syndrome in humans that results from loss of TASP1.

\section{Results}

To investigate the physiological function of Taspase1, we generated Tasp1-deficient mice (Tasp $1^{-/-}$mice). Our initial report demonstrated that these animals displayed marked homeotic transformation of the axial skeleton and a decrease in overall body size (11). Furthermore, Tasp $1^{-/-}$mice die shortly after birth, partly due to feeding defects (11). Here, we further examined the role of Taspase 1 in embryogenesis and initiated studies by comparing these Tasp $1^{-/-}$and WT littermate embryos. Tasp $1^{-/-}$E14.5 embryos show a severe developmental retardation, reflected by an approximately $40 \%$ reduction in overall weight in comparison to that of WT embryos (Figure 1A). Furthermore, Tasp $1^{-/-}$embryos have markedly smaller fetal livers (Figure 1B). The decrease in fetal liver cell number was significant even after normalizing for reduced body weight $(P<0.001)$ (Figure 1A).

We next analyzed the fetal liver for mature blood cell lineages and the hematopoietic stem and progenitor cell compartments using multicolor flow cytometry. The analysis of E14.5 fetal livers showed that Tasp $1^{-1}$ and WT fetal livers had little difference in the populations of B220+ $\mathrm{B}$ cells, Gr- $1^{+}$granulocytes, and TER-119+ red blood cells (Figure 1C). In contrast, the stem and progenitor cell populations, marked by Lin-Sca- $1^{+} \mathrm{C}-$ $\mathrm{Kit}^{+}$staining (so-called LSK cells), were reduced in Tasp $1^{-/-}$fetal livers (Figure 1, D and E). LSK cells can be further specified as multipotent progenitor cells (MPPs; $\mathrm{Lin}^{-} \mathrm{Sca}-1^{+} \mathrm{c}-\mathrm{Kit}^{+} \mathrm{CD} 150^{-}$) or hematopoietic stem cells (HSCs; $\left.\mathrm{Lin}^{-} \mathrm{Sca}-1^{+} \mathrm{c}-\mathrm{Kit}^{+} \mathrm{CD} 150^{+}\right)(24,25)$. In Tasp1 ${ }^{-/-}$fetal livers, the abundance of HSCs was found to be reduced to approximately half that of the WT fetal liver (Figure 1E), resulting in an approximately $78 \%$ decrease in the absolute number of HSCs (Figure 1F). No difference in the frequency of $\mathrm{Lin}^{-} \mathrm{Sca}-\mathrm{1}^{-} \mathrm{C}-\mathrm{Kit}^{+}$ myeloid progenitors was observed, whereas absolute numbers of these cells were decreased. Specifically, in Tasp $1^{-1-}$ fetal livers, the relative abundance of common myeloid progenitors (CMPs), granulocyte-monocyte progenitors (GMPs), and megakaryocyte-erythrocyte progenitors (MEPs) was normal (Figure 1, D and E). Together, our data indicate that, in the mouse fetal liver, Taspase 1 may be required for the development and maintenance of the HSC compartment.

Accordingly, we investigated how Taspase1 loss leads to impaired fetal liver HSC renewal. Loss of Taspase 1 activity could lead to increased cell death and/or aberrant cell cycle control. Cell death analysis of LSK cells and HSCs showed no increase of apoptosis in Tasp $1^{-/-}$fetal livers (Figure 2A). On the other hand, cell cycle analysis of MPPs and HSCs yielded a marked difference between WT and Tasp1 ${ }^{{ }^{\prime-}}$ cells. Most notably staining of DNA and RNA with 7-AAD and pyronin Y, respectively, showed that more Tasp $1^{-/-}$fetal liver HSCs exited stem cell quiescence, with decreased number of cells in the $\mathrm{G}_{0}$ phase, whereas the majority of WT HSCs were in the $\mathrm{G}_{0}$ phase, with fewer cells at $\mathrm{G}_{1}$ and $\mathrm{S} / \mathrm{G}_{2} / \mathrm{M}$ (Figure 2 , B and $\mathrm{C}$ ). In contrast, Tasp $1^{-/-}$fetal liver MPPs displayed similar cell cycle profile as WT MPPs. Strikingly, the cell cycle profile observed of Tasp $1^{-/-}$fetal liver HSCs resembles that of MPPs (Figure 2B).

To elucidate by which substrate(s) cleavage of Taspase1 regulates fetal liver hematopoiesis, we first focused on the MLL family proteins MLL1 and MLL2. MLL1 is the best-characterized Taspase1 substrate, controls Hox and Cylclin gene expression, and plays a critical role in both fetal and adult hematopoiesis (26-28). Homozygous knockin of noncleavable (nc) Mll1 mutant alleles (Mll1nc/nc) at the endogenous Mll1 locus did not reduce the overall fetal liver cellularity (Figure 3A) but incurred a minor decrease in frequency and number of HSCs (Figure 3B). As published data suggest a partial redundancy between M111 and M112 (11), we analyzed E14.5 fetal livers of Mllinc/nc; $2^{n c / n c}$ embryos bearing homozygous knockin of nc Mll1 and nc Mll2 mutant alleles at their native genomic loci and did not detect reduced fetal liver cellularity or body weight (Figure 3C). Similarly, the reduced abundance or absolute number of HSCs in $M l l 1^{n c / n c} ; 2^{n c / n c}$ fetal livers was not statistically significant (Figure 3D).

TFIIA family proteins TFIIA $\alpha-\beta$ and ALF are the only bona fide mammalian Taspase1 substrates known thus far besides MLL1 and MLL2. As expression of ALF is restricted to the mammalian testis (29), 
A Body Weight

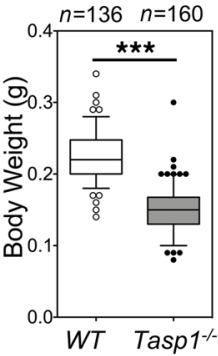

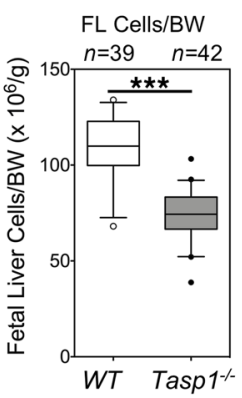

B

B $W T$

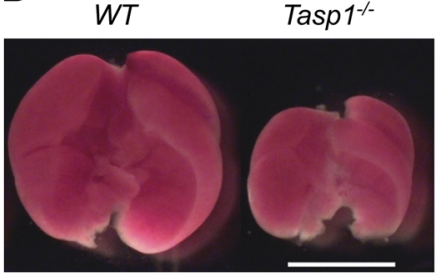

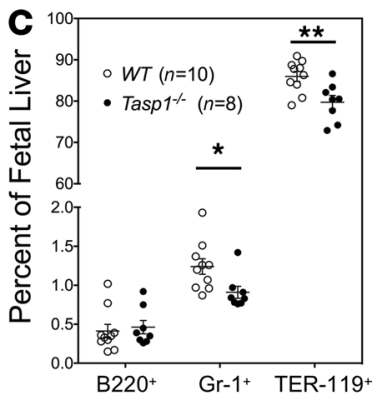

D
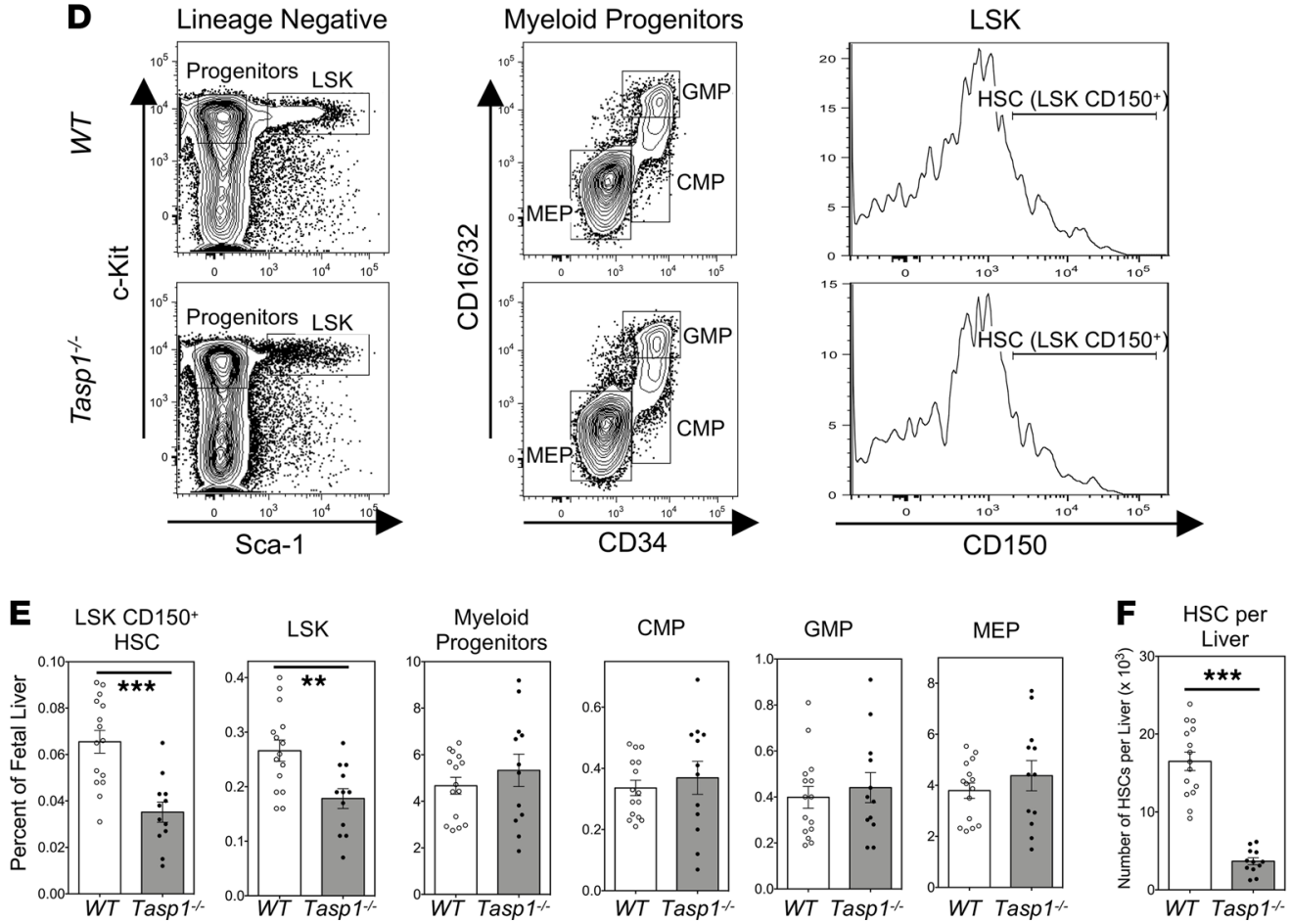

Figure 1. Taspase1 deficiency results in fetal liver hematopoietic stem cell defects. (A) Body weight and cell numbers of fetal livers (FLs) normalized to body weight of E14.5 embryos of the indicated WT and Taspase1-knockout (Tasp $1^{-/-}$) embryos. Boxes contain the 25 th to 75 th percentiles of data sets, with 50 th percentile center lines, and whiskers mark the 5th and 95th percentiles. Outliers are shown by dots. (B) Images of E14.5 FLs of the indicated genotypes. Scale bar: $2 \mathrm{~mm}$. (C) Frequency of E14.5 FL cells committed to erythroid (TER-119+), myeloid (Gr-1+), and B cell (B220+) lineages. (D) Progenitor and stem cell analyses of E14.5 FL cells by flow cytometry. Lin-Sca-1+c-Kit ${ }^{+}$cells are defined as LSK, and Lin-Sca-1'c$\mathrm{Kit}^{+}$cells as myeloid progenitors. Myeloid progenitors are subdivided into CMP, GMP, and MEP by CD34 and CD16/32. LSK CD150 ${ }^{+}$cells are defined as HSCs. (E) Quantification of stem and progenitor cells of 15 WT and 12 Tasp $^{1 /-}$ FLs. (F) HSCs per E14.5 FL of the indicated genotypes. Numbers were calculated by multiplying FL cellularity and HSC frequency. Data are shown as the mean \pm SEM. ${ }^{*} P<0.05,{ }^{* *} P<0.01,{ }^{* * *} P<0.001$ by Mann-Whitney $U$ test. HSC, hematopoietic stem cell; CMP, common myeloid progenitor; GMP, granulocyte-monocyte progenitor; MEP, megakaryocyte-erythrocyte progenitor.

we focused on the noncleavage of TFIIA $\alpha-\beta$. We have created a Gtf2a ${ }^{n c / n c}$ mouse model in which the endogenous $\mathrm{D} / \mathrm{G}$ cleavage residues of TFIIA $\alpha-\beta$ are replaced with noncleavable $\mathrm{A} / \mathrm{A}$ and have reported that Gtf2a $1^{n c / n c}$ males are infertile (22). Remarkably, Gtf2a $1^{n c / n c}$ embryos were smaller than their WT littermates, and their livers were disproportionally smaller (Figure 4, A and B). Furthermore, the frequencies of HSCs in Gtf2a1 ${ }^{n c / n c}$ fetal livers were also reduced (Figure 4D). In fact, the absolute HSC number of Gtf2a $1^{n c / n c}$ fetal livers was approximately $30 \%$ of that of their WT littermates (Figure 4E). Similar to Tasp $1^{-/-}$embryos, there were no significant changes in the frequency of myeloid progenitors, CMPs, GMPs, or MEPs in Gtf2a $1^{\text {nct } n c}$ fetal livers (Figure 4, C and D). Cell cycle analysis of Gtf2a $1^{n c / n c}$ fetal liver stem and progenitor cells demonstrated decreased a number of Gtf2a $1^{\text {nc/nc }} \mathrm{HSCs}$ in the $\mathrm{G}_{0}$ phase, which is reminiscent to that of WT and $G t f 2 a 1^{n c / n c}$ MPPs (Figure 5). The decrease in $\mathrm{G}_{0}$ phase and the coinciding increases in non- $\mathrm{G}_{0}$ phases of the 
A

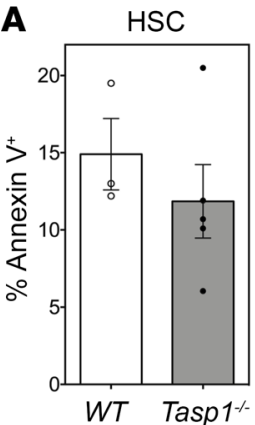

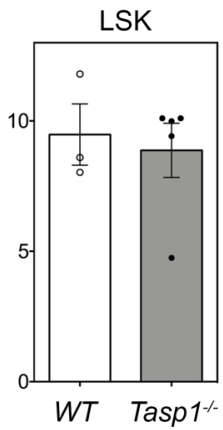

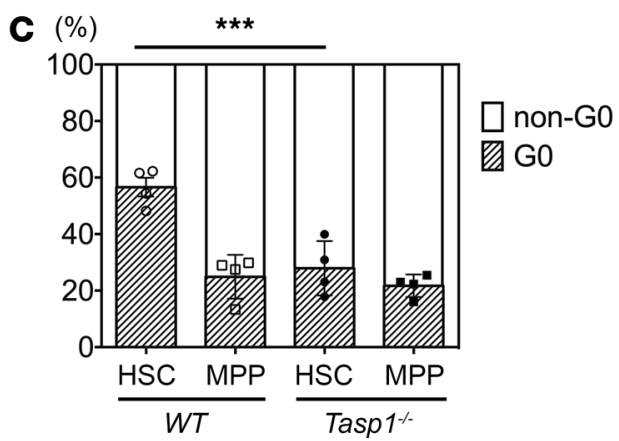

B

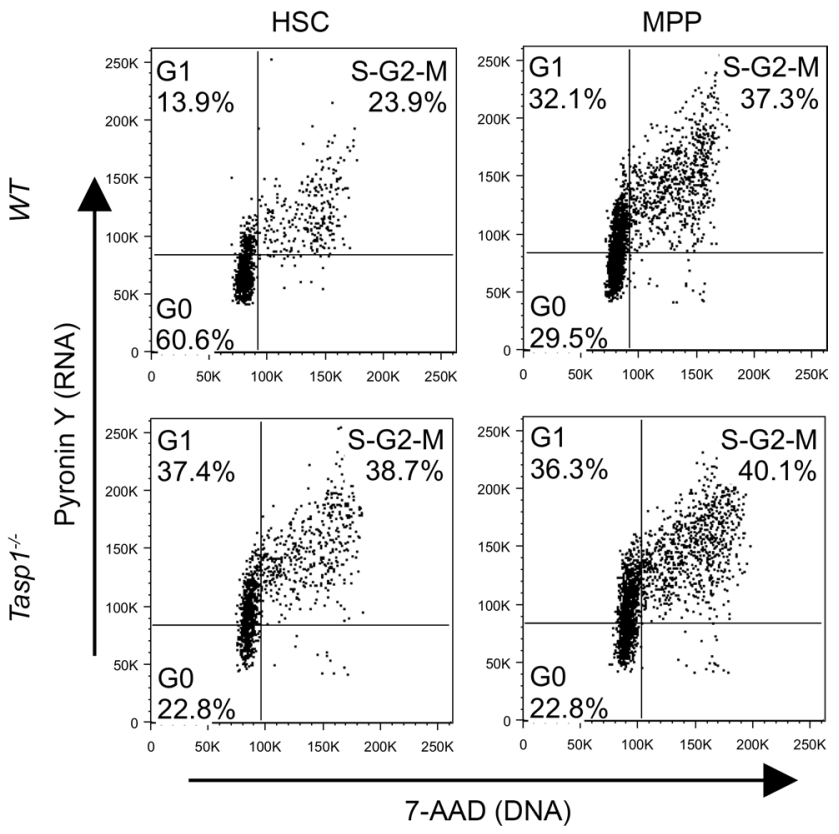

Figure 2. Tasp1-/- fetal liver hematopoietic stem cells exhibit an aberrant cell cycle profile reminiscent of multipotent progenitor cells. (A) Cell death analysis quantified by annexin $\mathrm{V}$ staining. The frequency of annexin $\mathrm{V}^{+}$cells in fetal liver (FL) hematopoietic stem cell (HSC) and LSK cells of the indicated genotypes was displayed (WT, $n=3 ;$ Tasp $^{1-1}, n=5$ ). Data are shown as the mean \pm SEM. (B and C) Cell cycle analysis of FL HSCs and multipotent progenitor cells (MPPs). Cells were stained with 7-AAD and pyronin $Y$ to assess DNA and RNA contents, respectively. (B) The quadrant gates of the representative plots based on DNA and RNA contents are outlined as $G_{0}, G_{1}$ and $S / G_{2} / M$ phases. (C) The frequency of HSCs and MPPs in $G_{0}$ and non $-G_{0}\left(G_{1}\right.$ and $\left.S / G_{2} / M\right)$ phases. Data are shown as the mean $\pm S E M\left(n=4\right.$ for all samples). $P$ values of 4 independent experiments by $\chi^{2}$ testing were combined by the Fisher's method; ${ }^{* *} P<0.001$.

Gtf2a $1^{n / n c}$ fetal liver HSCs indicated a stem cell quiescence defect similar to that observed in Tasp $1^{-/-}$fetal liver HSCs (Figure 2, B and C). These striking similarities between Gtf2a1 ${ }^{n c / n c}$ and Tasp $1^{-/-}$fetal livers highlight the importance of the Taspase1-TFIIA axis in the maintenance of fetal liver HSCs.

To further investigate this potentially novel regulation, we employed stem cell transplant assays to evaluate the capacity of Tasp $1^{-/-}$and Gtf2a $1^{n / n c}$ fetal liver HSCs in long-term hematopoietic reconstitution. Competitive repopulation assays were performed by transplanting $150 \mathrm{CD} 45.2^{+} \mathrm{HSC}$ from WT, Tasp $1^{-/-}$, or Gtf2a $1^{n / n c}$ E14.5 fetal livers along with CD $45.1^{+}$competitor cells into lethally irradiated CD $45.1^{+}$mice (Figure 6A). Twelve weeks after transplantation, peripheral blood was analyzed for the contribution of donor-derived HSCs to mature blood lineages, including $\mathrm{B} 220^{+}$for $\mathrm{B}$ cells, $\mathrm{CD} 3^{+}$for $\mathrm{T}$ cells, and $\mathrm{Gr}-1^{+} \mathrm{Mac}-1^{+}$for myeloid cells (Figure 6, B and C). More than 5\% of CD45.2+ donor-derived cells in all 3 lineages were detected in 13 of 19 mice transplanted with WT HSCs, whereas multilineage reconstitution was detected in neither the 9 mice transplanted with Tasp $1^{-/-}$HSCs nor the 8 mice transplanted with Gtf2a ${ }^{\text {nc/nc }}$ HSCs (Table 1). Thus, the Taspase1-TFIIA axis appears to be required for long-term reconstitution of hematopoietic cells from fetal livers, suggesting its role in long-term HSC self-renewal.

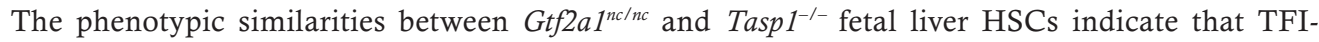
IA is the principal Taspase1 substrate conferring Taspase1-orchestrated fetal liver hematopoiesis. As noncleaved TFIIA $\alpha-\beta^{\text {nc }}$ is more stable than cleaved TFIIA $(5,22)$ and no apparent abnormalities were detected in Gtf2a $1^{n /+}$ fetal livers (data not shown), TFIIA $\alpha-\beta^{\text {nc }}$ is unlikely to function as a dominant-negative mutant in fetal liver hematopoiesis. Instead, data favors cleaved TFIIA positively regulating fetal liver hematopoiesis.

Hox genes of the vertebrates and homeotic genes of the invertebrates play critical roles in implementing body plan, and their deregulation results in the loss of segmental identities, i.e., homeotic transformation. As our previous studies demonstrated that Tasp $1^{-/-}$mice exhibit homeotic transformation (11), we examined the axial skeleton of $G t f 2 a 1^{n c / n c}$ newborn pups $(n=17)$ and detected overt homeotic 


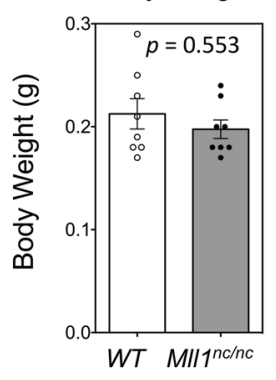

C Body Weight

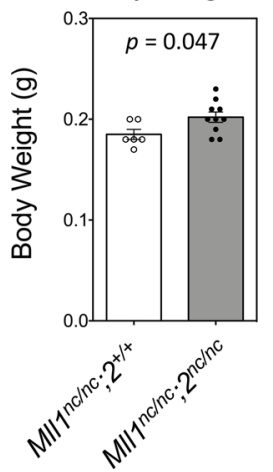

FL Cells/BW

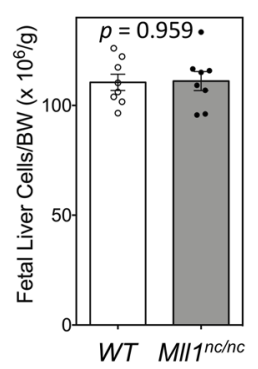

FL Cells/BW

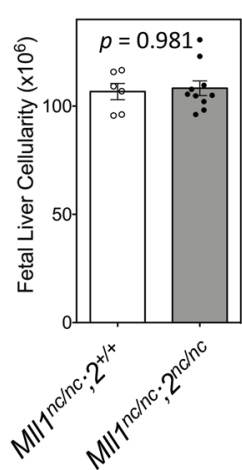

B
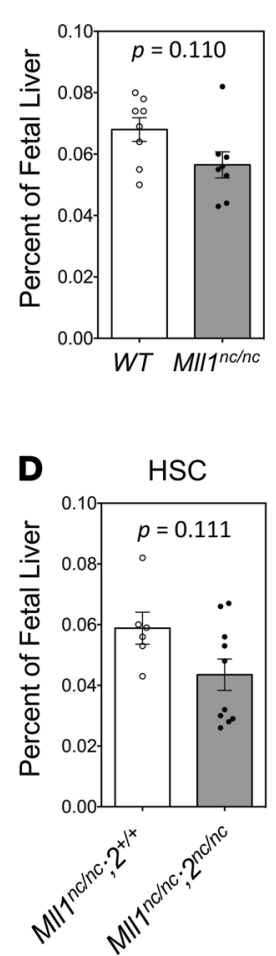
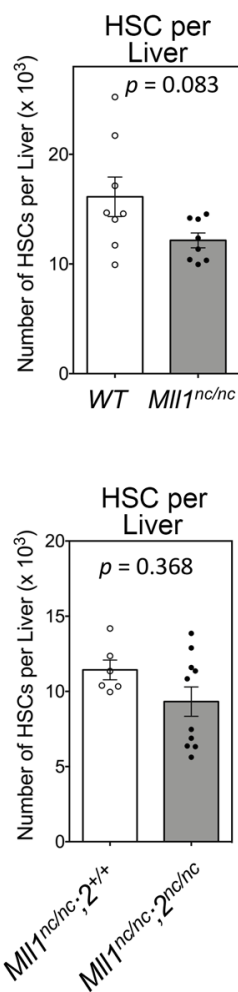

Figure 3. Hematopoietic stem cell quantification of $M I I 1^{n c / n c}$ and $M I I 1^{n c / n c} ; 2^{n c / n c}$ fetal livers. (A and B) Embryos obtained by intercrossing $\mathrm{MII1^{nc/+ }}$ mice were analyzed. (A) Body weight and normalized E14.5 fetal liver (FL) cellularity. (B) Fre-

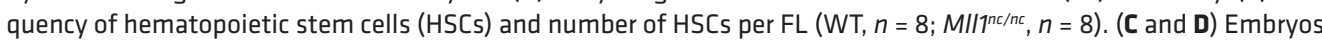
obtained by intercrossing MII/1n/nc; $2^{n c /+}$ mice were analyzed. (C) Body weight and normalized E14.5 FL cellularity. (D) Frequency of HSCs and number of HSCs per FL $\left(M I 11^{n c / n c} ; 2^{+/+}, n=6 ; M I 11^{n c / n c} ; 2^{n c / n c}, n=10\right)$. Data are shown as the mean \pm SEM. $P$ values were determined by Mann-Whitney $U$ test.

transformations, including abnormal anterior arch of atlas (a.a.a.) (82\%), split of C2 (cervical vertebra) (18\%), fusion of C3 to C5 (29\%), posterior transformation of C7 (65\%), anterior transformation of T8 (thoracic) (12\%), incomplete ossification of sternebra $4(65 \%)$, incomplete segmentation of sternebrae 3 and 4 (24\%), and posterior transformation of L6 (lumbar) (53\%) (Figure 7 and Table 2). Unexpectedly, homeotic defects of Gtf2a $1^{n c / n c}$ newborns were more profound than those of $M l l 1^{n c / n c} ; 2^{n c / n c}$ newborns and highly reminiscent of those of Tasp $1^{-/-}$newborns (Figure 7 and Table 2). Homeotic transformation of the axial skeletons is the defining feature of Hox gene deregulation $(30,31)$, and these extensive homeotic defects in Gtf2a1 ${ }^{n / n c}$ mice indicate a potentially novel regulation of Hox genes by TFIIA, a basal transcription factor, through a site-specific proteolytic process.

\section{Discussion}

Unlike most reversible posttranslational protein modifications, such as phosphorylation, acetylation, and methylation, proteolysis through either degradation or site-specific cleavage renders structural changes permanent and, thereby, potentially results in long-lasting functional consequences. In metazoans, site-specific proteolysis regulates critical aspects of biology, such as the activation of blood coagulation factors for hemostasis, the activation of caspases for cell death execution, the cleavage of Notch intracellular domain for cell fate determination, the release of SREBP for cholesterol homeostasis (32), the maturation of HCF and MLL1 for cell cycle progression $(11,12)$, and the assembly of mature TFIIA $\alpha / \beta / \gamma$ for male germ cell maturation $(5,22)$. Indeed, the identification and functional characterization of proteases and their cognate substrates have been instrumental in unraveling the underlying mechanisms concerning diverse biological processes.

Taspase 1 is a highly conserved protease that orchestrates a plethora of genetic programs through cleaving nuclear transcription regulators, MLL1, MLL2, TFIIA, ALF, and $\operatorname{dHCF}(6,33)$. Given that TFIIA is a basal transcription factor and Hox genes are highly specialized transcription factors, the 
A

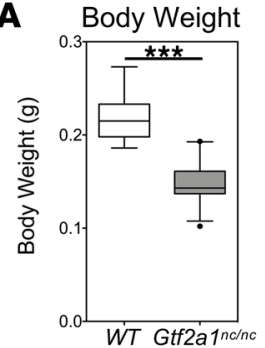

FL Cells/BW

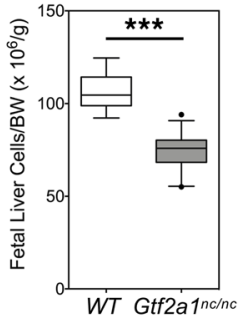

B

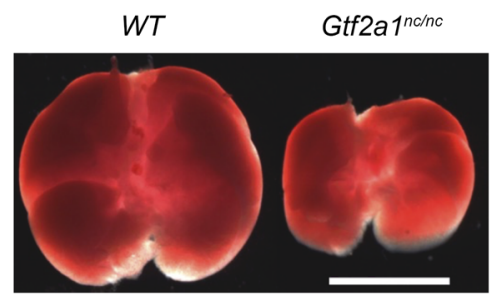

C

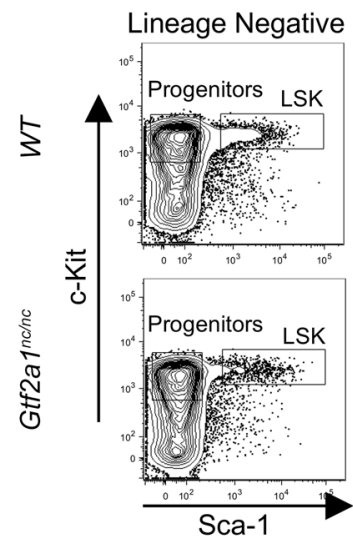

Myeloid Progenitors

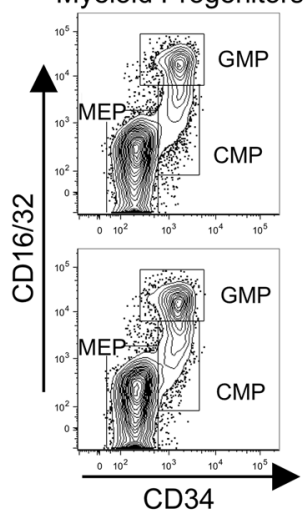

LSK
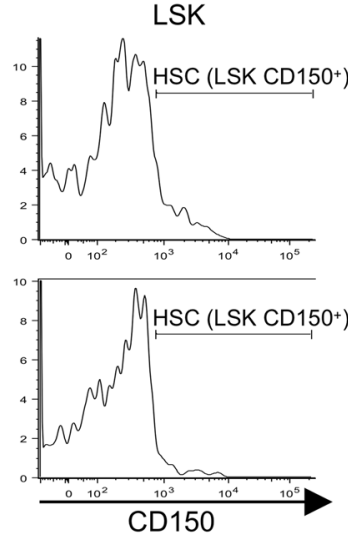

D
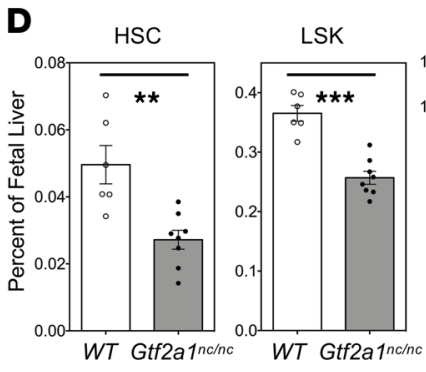

Myeloid Myeloid
Progenitors

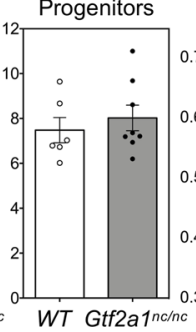

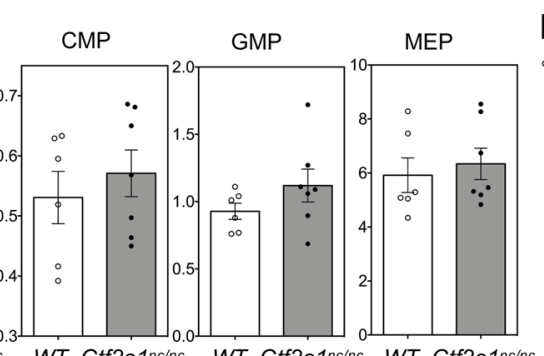

WT Gtf2a1nc/nc WT Gtf2a1nc/nc WT Gtf2a1nc/nc

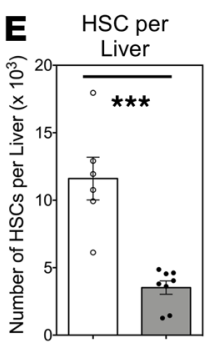

WT Gtf2a1nc/no
Figure 4. Gtf2a1nc/nc hematopoietic stem cells exhibit overt fetal liver hematopoiesis defects. (A) Body weight and normalized fetal liver (FL) cellularity of 19 WT and $27 \mathrm{Ctf} 2 a 1^{n / / n c}$ E14.5 FLs. Boxes contain the 25th to 75th percentiles of data sets, with 50 th percentile center lines, and whiskers mark the 5th and 95th percentiles. Outliers are shown by dots. ${ }^{* *} P<0.001$ by Mann-Whitney $U$ test. (B) Images of E14.5 FLs of the indicated WT and Gtf2a ${ }^{\text {nc/nc }}$ embryos. Scale bar: $2 \mathrm{~mm}$. (C and D) Quantification of hematopoietic stem and progenitor cells of E14.5 FLs by flow cytometry. (C) Representative dot plots. (D) The frequency of stem and progenitor cells in FLs. (E) The number of hematopoietic stem cells (HSCs) per FL. Data are shown as the mean \pm SEM. ${ }^{* *} P<0.01,{ }^{* *} P<0.001$ by Mann-Whitney $U$ test (WT, $n=$ 6; Gtf2a $a 7^{n c / n c}, n=8$ ).
Figure 5. Aberrant cell cycle profile of Gtf2a1nc/nc hematopoietic stem cells. Cell cycle analysis of Ctf2a ${ }^{n c / n c}$ E14.5 fetal liver $(\mathrm{FL})$ hematopoietic stem cells (HSC) and multipotent progenitor cells (MPPs). (A) The quadrant gates defining $G_{0}, G_{1}$, and $S / G_{2} / M$ phases. (B) The frequency of HSCs and MPPs in $G_{0}$ and non- $G_{0}\left(G_{1}\right.$ and $\left.S / G_{2} / M\right)$ phases. Data are shown as the mean $\pm \operatorname{SEM~}(n=4$ for all samples). $P$ values of 4 independent experiments by $\chi^{2}$ testing were combined by the Fisher's method; ${ }^{*} P<0.01$.

connections between Taspase1-mediated proteolytic processing of TFIIA and transcriptional regulation of Hox genes were completely unexpected, adding an additional layer of complexity to the intricate control of Hox gene expression through upstream transcription factors, epigenetic regulators, and long intergenic noncoding RNA $(34,35)$ for constructing segmental body plan and specifying cell lineages including stem cells.

A

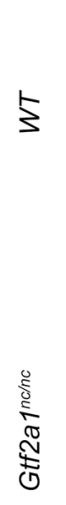

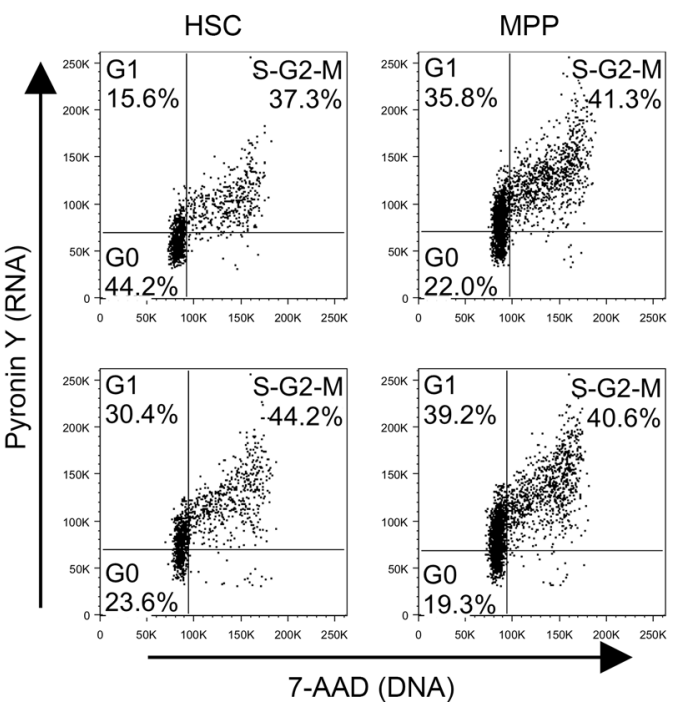

B

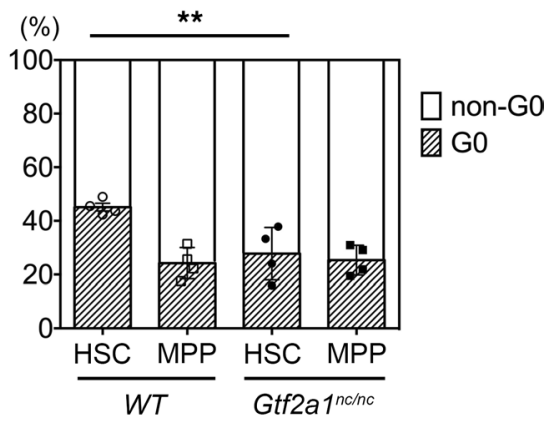


A

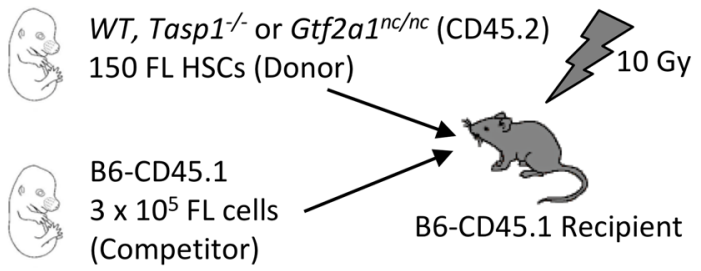

B
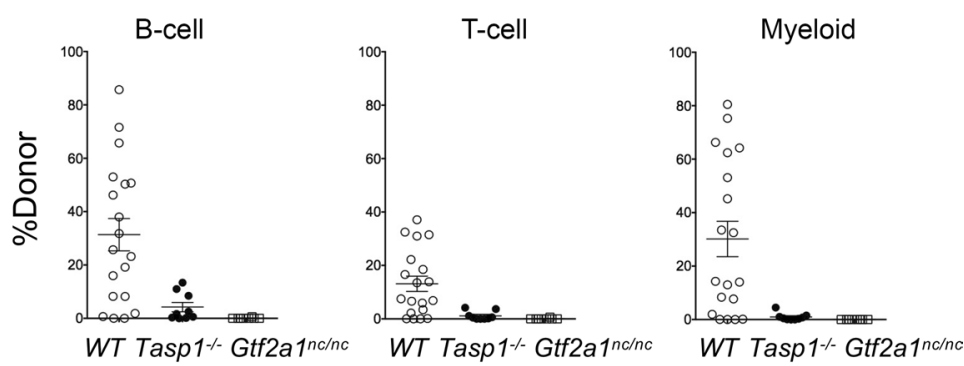

C

5

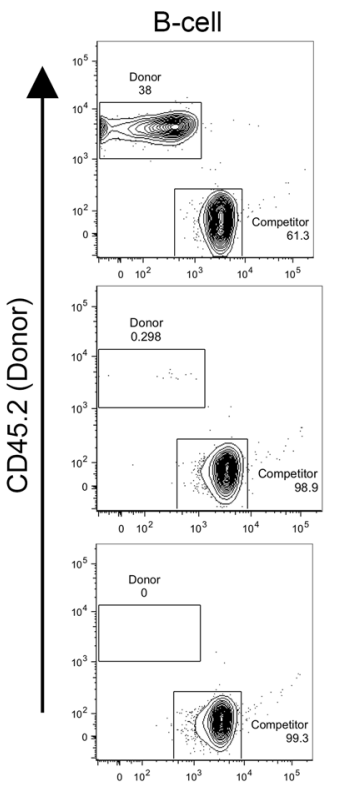

T-cell
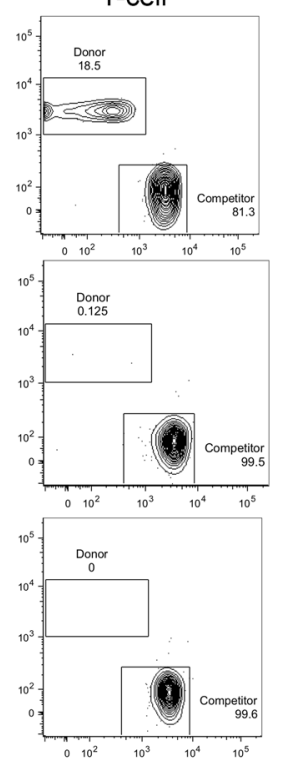

CD45.1 (Competitor)
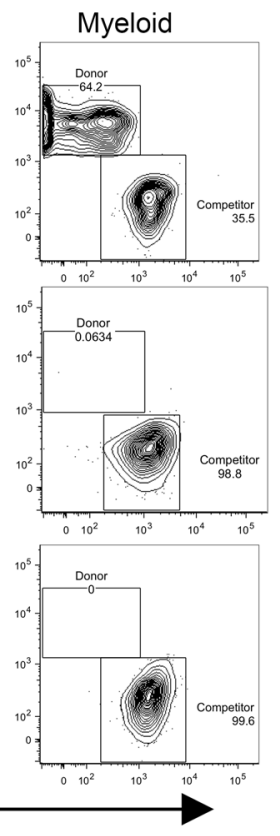

Figure 6. Tasp1 ${ }^{-/-}$and Ctf2a $1^{n / n c}$ fetal liver hematopoietic stem cells fail to reconstitute lethally irradiated recipient mice. (A) Outline of the experimental design of long-term competitive repopulation assays. (B) Scatterplots showing the frequency of donor-derived $B$ cell $\left(B 220^{+}\right), T$ cell $\left(\mathrm{CD}^{+}\right)$ and myeloid (Gr-1+Mac-1+ lineages reconstituted at 12 weeks after transplant. Data are shown as the average \pm SEM. WT, $n=19$; Tasp $^{-1-}, n=9$; Ctf2a1 ${ }^{n / / n c}$, $n=8$. (C) Representative plots show reconstitution by donor hematopoietic stem cells (HSCs) of the indicated genotypes in $B$ cell, $T$ cell, and myeloid lineages assessed 12 weeks after transplant. In each lineage, CD45.2+ and CD45.1 $1^{+}$cells were derived from donor (WT, Tasp1 ${ }^{1--}$ or Ctf2a $\left.a 7^{n / n c}\right)$ and competitor cells, respectively. See also Table 1.

The paradigm of Taspase1 function provides an opportunity to investigate how proteases interconnect diverse genetic programs via site-specific proteolysis. In the absence of Taspase1-mediated cleavage, MLL1, MLL2, and TFIIA $\alpha-\beta$ retain partial activity and animals bearing noncleaved MLL1, MLL2, and/or TFIIA $\alpha-\beta$ manifest hypomorphism in several biological settings (11, 22, 36, 37). Consequently, the alleles encoding nc MLL1, MLL2, and TFIIA $\alpha-\beta$ differ from null alleles and thus provide invaluable insight in cases where genetic ablation results in early embryonic lethality. Likewise, Taspase1 deficiency appears to offer a unique opportunity to uncover signaling pathways

Table 1. Multilineage reconstitution from WT, Tasp1 ${ }^{-/-}$, or Gtf2a $1 \mathrm{nc/nc}$ donor HSCs

\begin{tabular}{cc}
\hline Donor HSC genotype & Multilineage reconstitution \\
WT & $13 / 19$ \\
$T a s p 1^{-/-}$ & $0 / 9^{A}$ \\
$C t f 2 a 1^{n c / n c}$ & $0 / 8^{B}$
\end{tabular}

The number of mice in which multilineage reconstitution was detected per group is shown. ${ }^{A} P<0.001,{ }^{B} P<0.01$ by the Fisher's exact test (versus WT). HSCs, hematopoietic stem cells. 

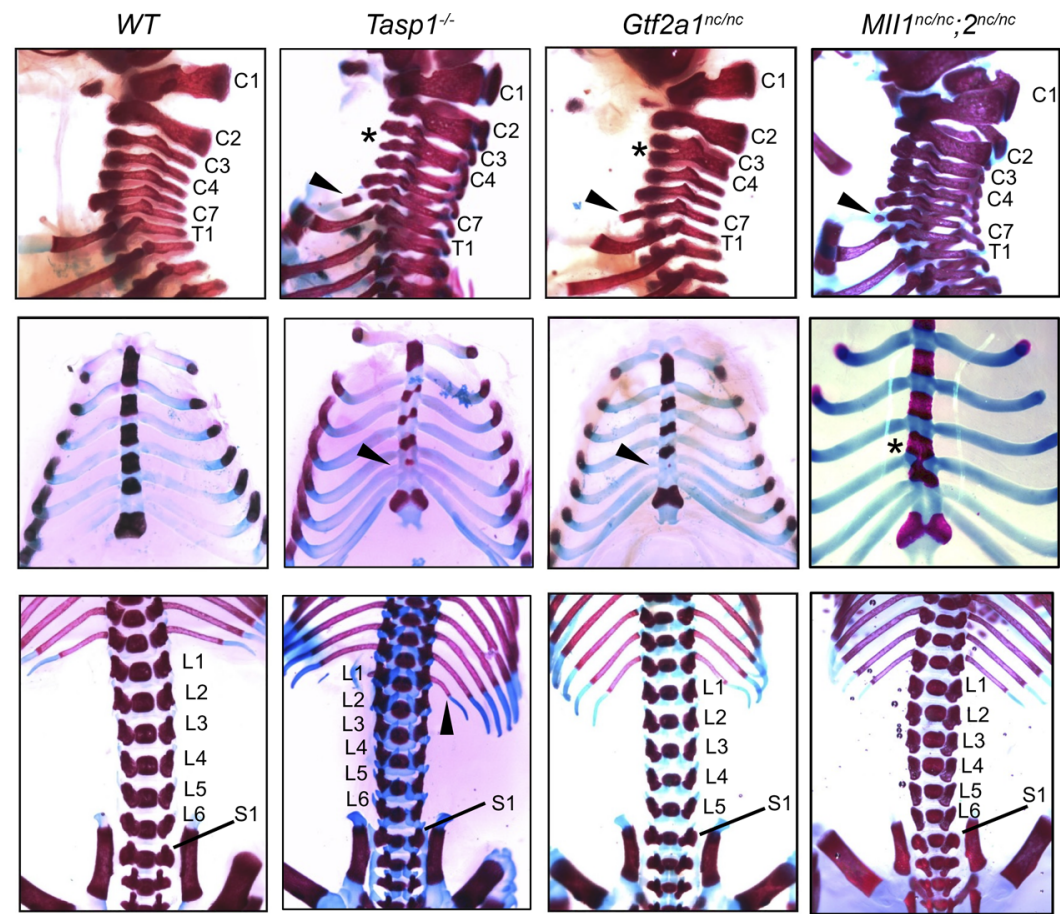

Figure 7. Homeotic transformation of axial skeletons in Tasp1-1- Gtf2a $1^{n c / n c}$, and $M I I 1^{n c / n c} ; 2^{n c / n c}$ newborns. Lateral views of cervical (C1-C7) and upper thoracic (T1) regions demonstrate deformed anterior arch of atlas (C1) and fusion

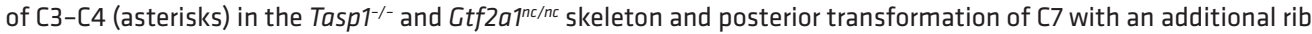
(arrowheads) in Tasp1 $1^{-/-}, G t f 2 a 7^{n c / n c}$, and $M I I 1^{n c / n c} ; 2^{n c / n c}$ skeleton (top row). Anterior views of the chest indicate incomplete ossification of sternebrae 4 (arrowheads) in Tasp1 ${ }^{-1-}$ and $G t f 2 a 1^{n c / n c}$ newborns and incomplete segmentation of sternebrae 3-4 (asterisks) in MII/nc/nc $; 2^{n c / n c}$ newborns (middle row). Anterior views of lower thoracic and lumbar vertebrae (L1-L6) demonstrate anterior transformation of L1 with an additional rib (arrowhead) in Tasp1 1/- newborns and posterior transformation of L6 in Ctf2a 1 $^{\text {c/nc }}$ newborns. See also Table 2.

controlled by developmentally essential genes. Taspase1 regulates the cell cycle, axial skeletal formation, fetal HSC homeostasis, male germ cell development $(11,22,36,37)$, and craniofacial development (23). As TFIIA complexes with not only TBP but also TRF2 (a male germ cell-enriched TBP variant) and TRF3 (a vertebrate-specific TBP variant), the Taspase1-TFIIA axis is positioned to orchestrate the assembly of diverse tissue-specific or context-dependent transcription machinery that are crucial for organismal development.

\section{Methods}

Mice. Tasp1 $1^{-1-}$, Mll1 $1^{n c / n c}, M l 2^{n c / n c}$, and Gtf2a $1^{n c / n c}$ mice have been previously described $(11,22)$. Tasp $1^{+/-}$and Gtf2a $1^{n c /+}$ mice were backcrossed to the WT C57BL6/J strain for 6 generations. Mll1 ${ }^{n c / n c}$ and Mll2 $2^{n c / n c}$ mice were similarly backcrossed to the WT C57BL6/J strain for 10 generations.

Flow cytometric analyses of hematopoietic cells. To obtain single-cell suspensions of hematopoietic cells, fetal livers were removed from E14.5 mouse embryos and homogenized by 3 passages through a 21-gauge needle. Freed cells were filtered through a nylon mesh with a pore size of $40 \mu \mathrm{m}$. Collected cells were treated with Red Blood Cell Lysing Buffer (MilliporeSigma) and then washed and filtered through nylon mesh. Automated blood cell counting was performed using a Hemavet Blood Analyzer (Drew Scientific). For mature blood cell analyses, 1 million cells were stained using the following antibodies: B220 APC (RA3-6B2) (BD Biosciences), Gr-1 PE (RB6-8C5) (BD Biosciences), and TER-119 Pacific Blue (TER-119) (BioLegend). For analyses of stem and progenitor cells, 1 million cells were stained using a combination of following antibodies: Lin (lineage antibody cocktail from BioLegend: CD3 [17A2], CD4 [GK1.5], CD8a [53-6.7], B220 [RA3-6B2], TER-119 [TER-119], and Gr-1 [RB6-8C5]) Pacific Blue (BioLegend), Sca-1 PE (D7) (BD Biosciences), c-Kit APC/Alexa Fluor 750 (2B8) (BD Biosciences), CD34 FITC (RAM34) (BD Biosciences), CD16/32 Alexa Fluor 647 (clone 93) (eBioscience), and CD150 PE/Cy7 (TC15-12F12.2) (BioLegend). To identify and exclude dead cells, 7-AAD was added in the final suspension. Cells positive 
Table 2. Skeletal defects observed in Tasp1 $1^{-/-}$, Gtf2a1 $1^{n c / n c}$, and $M I I 1^{n c / n c} ; 2^{n c / n c}$ newborns

\begin{tabular}{|c|c|c|c|c|}
\hline Skeletal defects & WT $(n=14)$ & $\operatorname{Tasp1}^{-/-}(n=17)$ & $C t f 2 a 1^{n c / n c}(n=17)$ & $M L L 1^{n c / n c} ; 2^{n c / n c}(n=10)$ \\
\hline a.a.a., tilted, broadened, and deformed & $14 \%(2)$ & $47 \%(8)$ & $82 \%(14)$ & $20 \%(2)$ \\
\hline C2, split neural arch & $0 \%(0)$ & $12 \%(2)$ & $18 \%(3)$ & $0 \%(0)$ \\
\hline C7, posterior transformation & $0 \%(0)$ & $94 \%(16)$ & $65 \%(11)$ & $50 \%(5)$ \\
\hline T8, anterior transformation & $0 \%(0)$ & $59 \%(10)$ & $12 \%(2)$ & $0 \%(0)$ \\
\hline Sternebrae 4, incomplete ossification & $0 \%(0)$ & $41 \%(7)$ & $65 \%(11)$ & $0 \%(0)$ \\
\hline L6, posterior transformation & $0 \%(0)$ & $0 \%(0)$ & $53 \%(9)$ & $0 \%(0)$ \\
\hline
\end{tabular}

a.a.a., abnormal anterior arch of atlas.

for Sca-1, CD34, or CD150 were determined by fluorescence-minus-one controls. Cells were analyzed with an LSRFortessa flow cytometer (BD Biosciences). Dot plots and histograms were made with FlowJo software. Graphs and statistical analyses were prepared with GraphPad Prism 6.

Sorting of hematopoietic stem and progenitor cells. Single-cell suspensions were prepared from fetal livers of E14.5 embryos or adult bone marrow and stained as described above. Fetal liver cells from multiple littermate embryos of the same genotype were pooled to ensure sufficient number of cells for staining. Stained cells were subsequently double sorted using a FACSAria II (BD Biosciences) to a final purity of more than $95 \%$.

Competitive reconstitution assay. C57BL6/J-CD45.1 mice were used as recipients and competitors. The recipient mice were irradiated by $10 \mathrm{~Gy}$ divided into 2 fractions on the day -1 . Competitor cells were prepared from E14.5 fetal livers from the C57BL6/J-CD45.1 mice and frozen in advance. On day 0, LSK CD $150^{+}$HSCs were sorted from E14.5 fetal livers of donor embryos that express CD45.2. Each recipient mouse was transplanted with 150 donor HSCs and $3 \times 10^{5}$ competitor fetal liver cells by tail vein injection. After 12 weeks, peripheral blood and bone marrow cells of recipients were analyzed for the presence of CD 45.1 $1^{+}$and CD $45.2^{+}$cells by a LSRFortessa. Peripheral blood mononucleic cells were stained with the following antibodies for analyzing myeloid lineage: CD45.1 PE (A20) (BD Biosciences), CD45.2 FITC (clone 104) (BD Biosciences), Mac-1 Ax647 (M1/70) (BD Biosciences), and Gr-1 Pacific Blue (RB6-8C5) (BioLegend). The following antibodies were used for lymphoid lineage: CD45.1 PE (A20), CD45.2 FITC (clone 104), B220 APC (RA3-6B2), and CD3 Pacific Blue (17A2).

Cell cycle analysis. Suspensions of sorted hematopoietic stem and progenitor cells were fixed with ethanol overnight at $4^{\circ} \mathrm{C}$, washed, and then resuspended in the house-made Nucleic Acid Staining Solution (38). Cells were stained with 7-AAD at room temperature for 20 minutes, followed by pyronin Y staining on ice for 15 minutes. Stained cells were analyzed by a LSRFortessa flow cytometer.

Annexin $V$ assay. One million fetal liver cells were stained with the following antibodies: Lin Pacific Blue, Sca-1 PE (D7), c-Kit APC/Ax750 (2B8), and CD150 PE/Cy7 (TC15-12F12.2). The cells were subsequently washed, stained with annexin V FITC (BioVision) and Propidium Iodide (MilliporeSigma), and then analyzed by flow cytometry.

Skeletal studies. P1 newborns were sacrificed and stained using Alizarin red (MilliporeSigma) and Alcian blue (MilliporeSigma) for bone and cartilage, respectively, as described previously (39).

Statistics. Statistical significance was evaluated using the Mann-Whitney $U$ test for continuous variables, unless otherwise specified. $\chi^{2}$ testing was performed on cell cycle analyses, and $P$ values of independent experiments were combined by the Fisher's method. $P$ values of less than 0.05 were considered significant.

Study approval. All animal work was performed in accordance to a protocol approved by the Institutional Animal Care and Use Committee of Memorial Sloan Kettering Cancer Center.

\section{Author contributions}

JJH designed the study. HN, ACS, and ST performed experiments and acquired and analyzed data. SAA, CYP, EHC, and JJH supervised experiments and analysis. HN and JJH wrote the manuscript. 


\section{Acknowledgments}

We apologize to all the investigators whose research could not be appropriately cited owing to space limitations. This work is supported by NIH grants CA119008 and CA138505 and a Scholar Award from the American Cancer Society to JJH.

Address correspondence to: Hidetaka Niizuma, Department of Pediatrics, Tohoku University School of Medicine, 1-1 Seiryo-machi, Sendai, Miyagi 980-8574, Japan. Phone: 81.22.717.7287; Email: hniizuma@med.tohoku.ac.jp. Or to: James J. Hsieh, Molecular Oncology, Department of Medicine, Washington University School of Medicine, 660 South Euclid Avenue, St. Louis, Missouri 63110 , USA. Phone: 314.454.8681; Email: jhsieh@wustl.edu.

1. Suleiman J, et al. TASP1 is deleted in an infant with developmental delay, microcephaly, distinctive facial features, and multiple congenital anomalies. Clin Genet. 2018;94(1):170-173.

2. Suleiman J, et al. Homozygous loss-of-function variants of TASP1, a gene encoding an activator of the histone methyltransferases KMT2A and KMT2D, cause a syndrome of developmental delay, happy demeanor, distinctive facial features, and congenital anomalies. Hum Mutat. 2019;40(11):1985-1992.

3. Balkin DM, et al. TASP1 mutation in a female with craniofacial anomalies, anterior segment dysgenesis, congenital immunodeficiency and macrocytic anemia. Mol Genet Genomic Med. 2019;7(9):e818.

4. Hsieh JJD, et al. Taspase1: a threonine aspartase required for cleavage of MLL and proper HOX gene expression. Cell. 2003;115(3):293-303.

5. Zhou H, et al. Uncleaved TFIIA is a substrate for taspase 1 and active in transcription. Mol Cell Biol. 2006;26(7):2728-2735.

6. Niizuma H, et al. Taspase 1: a protease with many biological surprises. Mol Cell Oncol. 2015;2(4):e999513.

7. Hsieh JJD, et al. Proteolytic cleavage of MLL generates a complex of N-and C-terminal fragments that confers protein stability and subnuclear localization. Mol Cell Biol. 2003;23(1):186-194.

8. Khan JA, et al. Crystal structure of human Taspase1, a crucial protease regulating the function of MLL. Structure. 2005;13(10):1443-1452.

9. Chen DY, et al. A pharmacologic inhibitor of the protease Taspase1 effectively inhibits breast and brain tumor growth. Cancer Res. 2012;72(3):736-746.

10. Høiby T, et al. Cleavage and proteasome-mediated degradation of the basal transcription factor TFIIA. EMBO J. 2004;23(15):3083-3091.

11. Takeda S, et al. Proteolysis of MLL family proteins is essential for taspase1-orchestrated cell cycle progression. Genes Dev. 2006;20(17):2397-2409.

12. Capotosti F, et al. Species selectivity of mixed-lineage leukemia/trithorax and HCF proteolytic maturation pathways. Mol Cell Biol. 2007;27(20):7063-7072.

13. Milne TA, et al. MLL targets SET domain methyltransferase activity to Hox gene promoters. Mol Cell. 2002;10(5):1107-1117.

14. Nakamura T, et al. ALL-1 is a histone methyltransferase that assembles a supercomplex of proteins involved in transcriptional regulation. Mol Cell. 2002;10(5):1119-1128.

15. DeJong J, Roeder RG. A single cDNA, hTFIIA/alpha, encodes both the p35 and p19 subunits of human TFIIA. Genes Dev. 1993;7(11):2220-2234.

16. Ma D, et al. Isolation of a cDNA encoding the largest subunit of TFIIA reveals functions important for activated transcription. Genes Dev. 1993;7(11):2246-2257.

17. Ozer J, et al. Molecular cloning of the small (gamma) subunit of human TFIIA reveals functions critical for activated transcription. Genes Dev. 1994;8(19):2324-2335.

18. Ranish JA, Hahn S. The yeast general transcription factor TFIIA is composed of two polypeptide subunits. J Biol Chem. 1991;266(29):19320-19327.

19. Yokomori K, et al. Drosophila TFIIA-L is processed into two subunits that are associated with the TBP/TAF complex. Genes Dev. 1993;7(11):2235-2245.

20. Malecová B, et al. Taspase1 processing alters TFIIA cofactor properties in the regulation of TFIID. Transcription. 2015;6(2):21-32.

21. Suzuki H, et al. TBP-like protein (TLP) interferes with Taspase1-mediated processing of TFIIA and represses TATA box gene expression. Nucleic Acids Res. 2015;43(13):6285-6298.

22. Oyama T, et al. Cleavage of TFIIA by Taspase1 activates TRF2-specified mammalian male germ cell programs. Dev Cell. 2013;27(2):188-200.

23. Takeda S, et al. Taspase1-dependent TFIIA cleavage coordinates head morphogenesis by limiting Cdkn2a locus transcription. J Clin Invest. 2015;125(3):1203-1214.

24. Kiel MJ, et al. SLAM family receptors distinguish hematopoietic stem and progenitor cells and reveal endothelial niches for stem cells. Cell. 2005;121(7):1109-1121.

25. Kim I, et al. Enhanced purification of fetal liver hematopoietic stem cells using SLAM family receptors. Blood. 2006;108(2):737-744.

26. Yagi H, et al. Growth disturbance in fetal liver hematopoiesis of Mll-mutant mice. Blood. 1998;92(1):108-117.

27. McMahon KA, et al. Mll has a critical role in fetal and adult hematopoietic stem cell self-renewal. Cell Stem Cell. 2007;1(3):338-345.

28. Jude CD, et al. Unique and independent roles for MLL in adult hematopoietic stem cells and progenitors. Cell Stem Cell. 2007;1(3):324-337.

29. Upadhyaya $\mathrm{AB}$, et al. Identification of a general transcription factor TFIIAalpha/beta homolog selectively expressed in testis. J Biol Chem. 1999;274(25):18040-18048. 
30. Mallo M, et al. Hox genes and regional patterning of the vertebrate body plan. Dev Biol. 2010;344(1):7-15.

31. Alexander T, et al. Hox genes and segmentation of the hindbrain and axial skeleton. Annu Rev Cell Dev Biol. 2009;25:431-456.

32. Brown MS, et al. Regulated intramembrane proteolysis: a control mechanism conserved from bacteria to humans. Cell. 2000;100(4):391-398

33. Stauber RH, et al. Cleaving for growth: threonine aspartase 1--a protease relevant for development and disease. FASEB J. 2016;30(3):1012-1022.

34. He H, et al. Epigenetic regulations in hematopoietic Hox code. Oncogene. 2011;30(4):379-388.

35. Wang KC, et al. A long noncoding RNA maintains active chromatin to coordinate homeotic gene expression. Nature. 2011;472(7341):120-124.

36. Dong Y, et al. Taspase1 cleaves MLL1 to activate cyclin E for HER2/neu breast tumorigenesis. Cell Res. 2014;24(11):1354-1366.

37. Takeda S, et al. HGF-MET signals via the MLL-ETS2 complex in hepatocellular carcinoma. J Clin Invest. 2013;123(7):3154-3165.

38. Toba K, et al. Simultaneous three-color analysis of the surface phenotype and DNA-RNA quantitation using 7-amino-actinomycin D and pyronin Y. J Immunol Methods. 1995;182(2):193-207.

39. Yu BD, et al. Altered Hox expression and segmental identity in Mll-mutant mice. Nature. 1995;378(6556):505-508. 